\title{
High Temperature Dynamic Pressure Measurements Using Silicon Carbide Pressure Sensors
}

\author{
Robert S. Okojie ${ }^{1}$, Roger D. Meredith ${ }^{1}$, Clarence T. Chang ${ }^{1}$, and Ender Savrun ${ }^{2}$ \\ ${ }^{1}$ NASA Glenn Research Center, M/S 77-1, 21000 Brookpark Road, Cleveland, OH 44135 \\ ${ }^{2}$ Sienna Technologies, Inc, 19501 144th Ave NE \# F500, Woodinville, WA 98072
}

\begin{abstract}
Un-cooled, MEMS-based silicon carbide (SiC) static pressure sensors were used for the first time to measure pressure perturbations at temperatures as high as $600{ }^{\circ} \mathrm{C}$ during laboratory characterization, and subsequently evaluated in a combustor rig operated under various engine conditions to extract the frequencies that are associated with thermoacoustic instabilities. One $\mathrm{SiC}$ sensor was placed directly in the flow stream of the combustor rig while a benchmark commercial water-cooled piezoceramic dynamic pressure transducer was co-located axially but kept some distance away from the hot flow stream. In the combustor rig test, the SiC sensor detected thermoacoustic instabilities across a range of engine operating conditions, amplitude magnitude as low as 0.5 psi at $585{ }^{\circ} \mathrm{C}$, in good agreement with the benchmark piezoceramic sensor. The SiC sensor experienced low signal to noise ratio at higher temperature, primarily due to the fact that it was a static sensor with low sensitivity.
\end{abstract}

Key words: SiC Pressure Sensor, Dynamic Test, and High Temperature.

\section{Introduction}

Dynamic pressure sensors are required to quantify pressure dynamics in the combustor chamber of jet and gas turbine engines. These dynamics may indicate an onset of thermoacoustic instabilities that could degrade engine performance. Also, the demand for lower emissions (LE) in aircraft gas-turbine engines has resulted in advanced combustor designs that are critically dependent on lean-burning (LB) operation. However, LB/LE combustors are susceptible to thermoacoustic instabilities that can produce large pressure oscillations within the combustor. This can at a minimum disrupt compressor flow or potentially lead to premature mechanical failures. A combustor is essentially an acoustic resonator. As an engine is run through its operating range, there are states where the heat release coupling with the acoustics forms unacceptably high pressure oscillations [1]. This problem is expected to be more pronounced in LB/LE combustion systems because less system damping is present with LB combustors due to reduced liner cooling. The reduced liner cooling is the result of the diversion of more cooling air to the combustion process. Engine ground tests have traditionally utilized miniature dynamic pressure sensors (microphones) to measure thermoacoustic instabilities. These sensors were either cooled or located at some stand-off distance from the 
combustor, which resulted in high background noise, acoustic propagation delays, as well as limiting the frequency range of the measurement. Potentially, optical sensors can give timely responses and cleaner signals [2], but would be more effective if they could resolve the events near the fuel injectors in the combustor front-end. Silicon based miniature pressure sensors are reliable up to $350^{\circ} \mathrm{C}$ with water cooling. Commercially available pressure sensors that utilize quartz crystals are specified to operate up to $400{ }^{\circ} \mathrm{C}$ without water cooling [3]. Therefore, in order to ensure measurement integrity during combustor condition diagnostics, these transducers are placed several inches away from the hot sections through an infinite loop tube. Such measurement strategy causes the attenuation of key frequency components and delay in pressure propagation, thus affecting signal fidelity. It also diminishes the opportunity for the signal to be applied for real time active control of combustion instabilities. The only known commercially available dynamic pressure sensor uses $\mathrm{GaPO}_{4}$ piezoelectric crystal reported to operate between 400 and $780^{\circ} \mathrm{C}[4,5]$. This material is synthesized, since it does not exist in nature, and there is only one known global manufacturer.

Miniaturized and un-cooled SiC pressure sensors are emerging as viable alternatives due to the high temperature operational capability, having been demonstrated to survive $600{ }^{\circ} \mathrm{C}$ operation and currently undergoing long term reliability assessment $[6,7]$. We recently reported the first demonstration of MEMS-based $4 \mathrm{H}-\mathrm{SiC}$ piezoresistive static pressure sensors operating at $750{ }^{\circ} \mathrm{C}$ and in the process confirmed that the sensor achieves $100 \%$ recovery of the full-scale output (FSO) at $750{ }^{\circ} \mathrm{C}$ [8]. This FSO recovery phenomenon in $4 \mathrm{H}-\mathrm{SiC}$ is uncharacteristic of the well-known monotonic decrease in strain sensitivity with increasing temperature found in silicon piezoresistors. This result will allow the operation of SiC pressure sensors at higher temperatures, thereby permitting deeper insertion into the engine combustion chamber to improve the accurate quantification of combustor dynamics.

In this work, a static SiC pressure sensor rated at 500 psig was benchmarked against a piezoceramic dynamic pressure sensor as a worse case performance assessment, while the process to implement an appropriate dynamic SiC pressure sensor continues.

\section{Sensor Pre-test Characterization}

For the purpose of pre-test calibration, a static characterization of the SiC pressure sensor was performed to extract calibration parameters such as the zero pressure offset voltage and full-scale output as function of temperature. The sensors used in these tests were rated to operate up to $600{ }^{\circ} \mathrm{C}$ and $500 \mathrm{psig}$. The accelerated stress test protocol used for the static characterization has been reported elsewhere [7]. Subsequently, a laboratory characterization of the static SiC pressure sensors and a benchmark water-cooled piezoceramic dynamic 
pressure sensor was performed to determine the former's ability to respond to pressure perturbations as function of temperature. The dynamic pressure generator system was setup to allow a steady stream of compressed air (typically about 100 psig) to flow through a seven foot long, 0.25 inch diameter, 316 stainless steel tubing, that was folded seven times so as to fit inside a horizontal tube furnace. The room temperature air thus flows through seven feet of folded tube in the furnace and in the process is heated to the desired temperature before exiting the furnace. The SiC pressure sensor is placed immediately at the furnace exit, with a thermocouple to monitor the exiting hot air temperature. Aft of the SiC sensor, the watercooled reference piezoceramic pressure sensor is placed to monitor the air stream continuing out the tube. Further downstream, a flow-restricting orifice reduces the inner diameter of the 0.250 " tube down to 0.031 " diameter before the open end of the tube. A high-speed piezoelectric pulse valve was teed in the line in front of the exit orifice. Thus, closing the open end of the tube will cause the pressure to rise inside the tube to a static value of 100 psig. While at this state, the valve was actuated with a pre-selected transient excitation voltage (i.e., sinusoidal), which would alternately open and close the valve, thereby causing correspondingly alternating pressure rises and drops within the tube, which were sensed and measured by the $\mathrm{SiC}$ and piezoceramic pressure sensors.
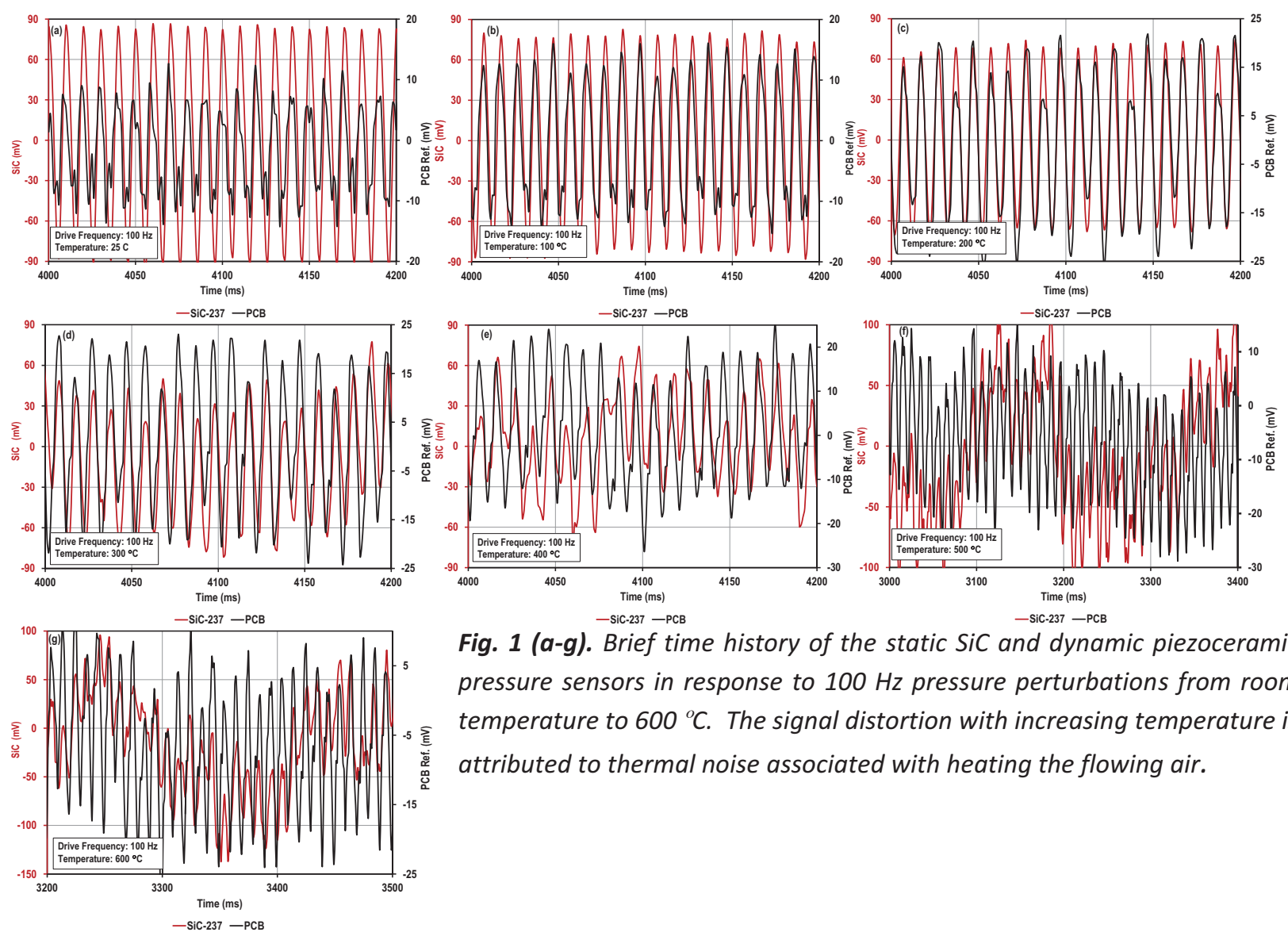

Fig. 1 (a-g). Brief time history of the static SiC and dynamic piezoceramic pressure sensors in response to $100 \mathrm{~Hz}$ pressure perturbations from room temperature to $600{ }^{\circ} \mathrm{C}$. The signal distortion with increasing temperature is attributed to thermal noise associated with heating the flowing air. 
The response of the un-cooled $\mathrm{SiC}$ and water-cooled pressure sensors to an applied $100 \mathrm{~Hz}$ pulse from room temperature to $600{ }^{\circ} \mathrm{C}$ is shown in Figs. 1 (a-g). From room temperature to $200{ }^{\circ} \mathrm{C}$ (Figs. 1a-c), the two sensors responded well to the $100 \mathrm{~Hz}$ perturbations. However, starting from $300{ }^{\circ} \mathrm{C}$ (Figs. d-g), the signals of both sensors become gradually more distorted, likely due to thermal turbulence noise effect from the heated air. The SiC sensor response, however, continues to track that of the piezoceramic sensor despite the increasing distortion with increasing temperature. In addition to the noise effect, the peak-peak response of the SiC sensor also gradually drops with increasing temperature. The Fast Fourier Transform amplitude response is shown in Figs 2 (a) and (b) for the static SiC and piezoceramic pressure sensors, respectively. Both show response to the $100 \mathrm{~Hz}$ pressure perturbation. The two spectral peaks in the piezoceramic sensor are suspected to be due to the thermal noise stated earlier.


Fig. 2: Amplitude magnitude response of the, a) static SiC pressure sensor and, b) the dynamic piezoceramic pressure sensor at $100 \mathrm{~Hz}$ as function of temperature during laboratory characterization with the dynamic pressure generator.

\section{Combustor rig test}

Following laboratory characterization, the SiC pressure sensor was inserted into the combustor test rig, flush mounted with the inner liner, thereby allowing direct interaction with the flow stream. Another benchmark water-cooled piezoceramic sensor was co-located axially with the SiC pressure sensor, but recessed away from the flow stream. The combustor was then operated through various engine conditions (temperature, pressure, and fuel/air ratio) to identify operating points that were associated with thermoacoustic instabilities. The response of the SiC pressure sensor was then compared with the reference water-cooled piezoceramic sensor. Figures 3 (a) and (b) show the comparisons between the $\mathrm{SiC}$ and piezoceramic sensors in terms of thermoacoustic frequencies and amplitude response to various combustion conditions. The amplitude response after ignition revealed frequencies that were associated with thermoacoustic instabilities under various operating conditions. The thermoacoustic frequencies and their

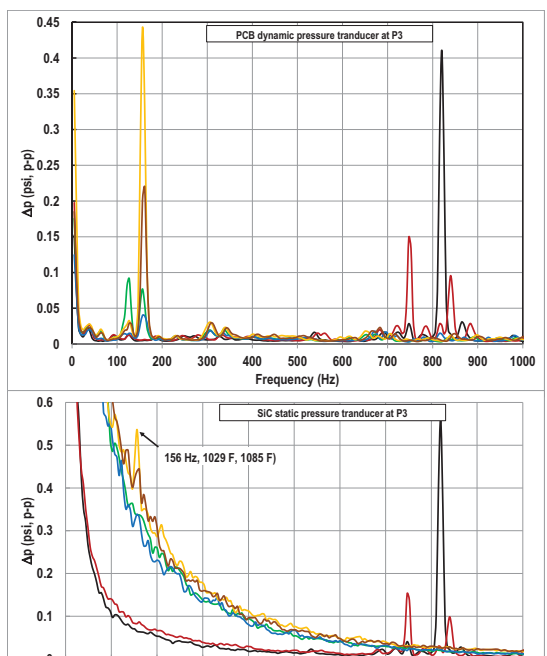

Fig. 3. Amplitude spectral density for the a) piezoceramic pressure sensor and b) SiC pressure sensor both axially co-located at the P3 section of the combustor rig. They both detected thermoacoustic instabilities across various test conditions. amplitude responses detected by the two sensors at lower operating temperatures were in 
excellent agreement, detecting magnitudes as low as $0.1 \mathrm{psi}$ at lower temperatures. However, the $\mathrm{SiC}$ sensor could not resolve with fidelity the amplitudes and frequencies at the three higher operating temperatures due to thermal noise that reduced the signal/noise ratio. It should be noted that the $\mathrm{SiC}$ sensors used here were designed for static pressure applications with rating of 500 psig. As a result, the thermal noise introduced at higher temperatures resulted in a low signal/noise ratio. This shortcoming will be addressed by designing and fabricating a SiC dynamic pressure sensor with higher sensitivity.

\section{Conclusion}

An uncooled $4 \mathrm{H}-\mathrm{SiC}$ pressure transducer has demonstrated the ability to detect thermoacoustic instabilities at frequencies that are associated with thermoacoustic instabilities in a combustion test rig operating across various test conditions (temperature, pressure, fuel/air ratio). The results show excellent agreement with a benchmark water-cooled piezoceramic pressure transducer that was axially co-located with the SiC pressure sensor. Due to its flexible packaging, lightweight, relatively low cost, and robustness to survive higher temperature and vibration beyond conventional transducers, the SiC pressure transducer offers proximal detection of thermoacoustic instabilities in aero-engines with accuracy that is comparable with a water-cooled piezoceramic pressure sensor.

\section{Acknowledgement}

The funding for this work was provided by the NASA Environmentally Responsible Aviation and Aeronautical Sciences Projects under the Integrated Systems Research and Fundamental

Aeronautics Programs, respectively. The authors thank the NASA Glenn Research Center Combustor Branch for providing access and supporting the combustor rig tests.

\section{References}

[1] A.H. Lefebvre, "Gas Turbine Combustion", 2nd edition, Taylor \& Francis, 1999.

[2] M. Thiruchengode, S. Nair, Y. Neumeier, T. Lieuwen, J. Seitzman and B. Zinn, "An Active Control System for LBO Margin Reduction in Turbine Engines," 41st Aerospace Sciences Meeting \& Exhibit, Reno, Nevada, Jan. 69, 2003. AIAA-2003-1008.

[3] http://www.pcb.com/TestMeasurement/Pressure/Extreme_Environ.aspx.

[4] https://www.avl.com/c/document_library

[5] http://www.vibro-meter.com/pdf/Cp215e.pdf.

[6] R. S. Okojie, G. M. Beheim, G. J. Saad, and E. Savrun, "Characteristics of a Hermetic 6H-SiC Pressure Sensor at $600{ }^{\circ} \mathrm{C}$," 39th Aerospace Sciences Meeting \& Exhibit, Reno, Nevada, Jan. 8-11, 2001. AIAA 2001-4652. 
[7] R. S. Okojie, E. Savrun, P. Nguyen, V. Nguyen, and C. Blaha, "Reliability Evaluation of Direct Chip Attached SiC Pressure Transducers," Proc. IEEE Sensors Conference, Vienna, Austria, October 24-27, 2004, pp. 635638(2004).

[8] This conference. 